\title{
Three-Dimensional Ordered-Subset Expectation Maximization Iterative Protocol for Evaluation of Left Ventricular Volumes and Function by Quantitative Gated SPECT: A Dynamic Phantom Study
}

\author{
Luca Ceriani $^{1}$, Teresa Ruberto ${ }^{1}$, Angelika Bischof Delaloye ${ }^{2}$, John O. Prior ${ }^{2}$, and Luca Giovanella ${ }^{1}$ \\ ${ }^{I}$ Department of Nuclear Medicine and PET/CT Centre, Oncology Institute of Southern Switzerland (IOSI), Bellinzona, Switzerland; and \\ ${ }^{2}$ Department of Nuclear Medicine, Center Hospitalier Universitaire Vaudois (CHUV) and University of Lausanne, Lausanne, \\ Switzerland
}

\begin{abstract}
The purposes of this study were to characterize the performance of a 3-dimensional (3D) ordered-subset expectation maximization (OSEM) algorithm in the quantification of left ventricular (LV) function with $99 \mathrm{mTc}$-labeled agent gated SPECT (G-SPECT), the QGS program, and a beating-heart phantom and to optimize the reconstruction parameters for clinical applications. Methods: A G-SPECT image of a dynamic heart phantom simulating the beating left ventricle was acquired. The exact volumes of the phantom were known and were as follows: end-diastolic volume (EDV) of $112 \mathrm{~mL}$, end-systolic volume (ESV) of $37 \mathrm{~mL}$, and stroke volume (SV) of $75 \mathrm{~mL}$; these volumes produced an LV ejection fraction (LVEF) of $67 \%$. Tomographic reconstructions were obtained after 10-20 iterations $(I)$ with 4,8 , and 16 subsets $(S)$ at full width at half maximum (FWHM) gaussian postprocessing filter cutoff values of $8-15 \mathrm{~mm}$. The QGS program was used for quantitative measurements. Results: Measured values ranged from 72 to $92 \mathrm{~mL}$ for EDV, from 18 to $32 \mathrm{~mL}$ for ESV, and from 54 to 63 $\mathrm{mL}$ for SV, and the calculated LVEF ranged from $65 \%$ to $76 \%$. Overall, the combination of $10 I, 8 \mathrm{~S}$, and a cutoff filter value of $10 \mathrm{~mm}$ produced the most accurate results. The plot of the measures with respect to the expectation maximization-equivalent iterations $(I \times S$ product) revealed a bell-shaped curve for the LV volumes and a reverse distribution for the LVEF, with the best results in the intermediate range. In particular, FWHM cutoff values exceeding $10 \mathrm{~mm}$ affected the estimation of the LV volumes. Conclusion: The QGS program is able to correctly calculate the LVEF when used in association with an optimized 3D OSEM algorithm (8 S, $10 I$, and FWHM of $10 \mathrm{~mm}$ ) but underestimates the LV volumes. However, various combinations of technical parameters, including a limited range of $I$ and $S$ (80-160 expectation maximization-equivalent iterations) and low cutoff values $(\leq 10 \mathrm{~mm})$ for the gaussian
\end{abstract}

Received Sep. 3, 2009; revision accepted Dec. 28, 2009.

For correspondence or reprints contact: Luca Ceriani, Department of Nuclear Medicine and PET/CT Centre, Oncology Institute of Southern Switzerland, Via Ospedale 12, CH-6500 Bellinzona, Switzerland.

E-mail: luca.ceriani@eoc.ch

COPYRIGHT ( 2010 by the Society of Nuclear Medicine, Inc. postprocessing filter, produced results with similar accuracies and without clinically relevant differences in the LV volumes and the estimated LVEF.

Key Words: iterative protocols; OSEM; gated SPECT; beating cardiac phantom; QGS

J Nucl Med Technol 2010; 38:18-23

DOI: 10.2967/jnmt.109.070243

$\mathbf{I}$ terative reconstruction algorithms were recently proposed as a new potential tool for increasing the quality of tomographic images in nuclear medicine. Protocols based on ordered-subset expectation maximization (OSEM) have become the dominant iterative reconstruction procedures in SPECT and PET. The performance of OSEM was compared with that of filtered backprojection (FBP) in several experimental and clinical studies (1-5); OSEM was shown to have better noise properties and higher image contrast and resolution. On the other hand, even though iterative protocols were suggested for cardiac SPECT in the recent European Association of Nuclear Medicine/European Society of Cardiology guidelines (6), controversial results were reported when myocardial perfusion and left ventricular (LV) function were assessed by different iterative methods (maximumlikelihood expectation maximization, iterative-weighted, and 2-dimensional OSEM) (7-16). Standardization of these protocols has not been proposed yet.

The aims of this study were to characterize, with a beating-heart phantom having known volumes, the performance of a 3-dimensional (3D) OSEM algorithm with 3D gaussian postreconstruction filtering for the quantification of LV function by ${ }^{99 \mathrm{~m} T c-l a b e l e d ~ a g e n t ~ g a t e d ~ S P E C T ~}$ (G-SPECT) with the QGS program (Cedars-Sinai Medical Center) and various numbers of subsets $(S)$, iterations $(I)$, 
and filter cutoff values and to optimize the parameters for clinical applications.

\section{MATERIALS AND METHODS}

The G-SPECT acquisition of a dynamic heart phantom simulating the beating left ventricle (BSI) was obtained at the Department of Nuclear Medicine, Center Hospitalier Universitaire Vaudois and University of Lausanne, Lausanne, Switzerland. The dynamic heart phantom is part of an anthropomorphic torso phantom and comprises a membrane unit and a pump-control unit. In the membrane unit, the left ventricle of the heart is anatomically simulated by a double elastic membrane that, because of its position, has 2 compartments; the inner one, filled with saline solution, represents the ventricular cavity, and the outer one, enclosed between the 2 flexible silicone membranes, represents the heart wall. The external pump-control unit ensures controlled compression and decompression of the interior membrane, providing a change in the volume of the inner compartment. The pumping frequency is adjustable up to 70 cycles per minute. The pumping event simulates the cardiac cycle, and an appropriate electrocardiographic (ECG) trigger signal is generated. The volumes of the cardiac phantom, as provided by the manufacturer, are as follows: end-diastolic volume (EDV), $112 \mathrm{~mL}$; end-systolic volume (ESV), $37 \mathrm{~mL}$; and stroke volume (SV), $75 \mathrm{~mL}$. The corresponding value for the LV ejection fraction (LVEF) is $67 \%$ (Fig. 1]).

The heart wall chamber of the dynamic heart phantom was filled with $30 \mathrm{MBq}$ of ${ }^{99 \mathrm{~m}} \mathrm{Tc}$. It was imaged by use of a 2-head $\gamma$-camera (ECAM; Siemens Medical Systems, Inc.) in $90^{\circ}$ geometry and equipped with a low-energy high-resolution parallel-hole collimator. Sixty-four images were obtained from 32 angular positions with a $64 \times 64$ matrix (pixel dimension, $6.59 \mathrm{~mm}$ ) and an acquisition time of $40 \mathrm{~s}$ per step throughout a $180^{\circ}$ arc beginning from the $45^{\circ}$ right anterior oblique projection and ending at the $45^{\circ}$ left posterior oblique projection. The ECG G-SPECT acquisition was performed at a heartbeat frequency of 60 beats per minute with 8 frames per RR interval at each angular step. The protocol was the same as that used in our clinical applications, but the acquisition time was increased and tailored to collect from the cardiac area several counts similar to those routinely obtained in patients (1.5-2.8 kilocounts per frame for the different angular

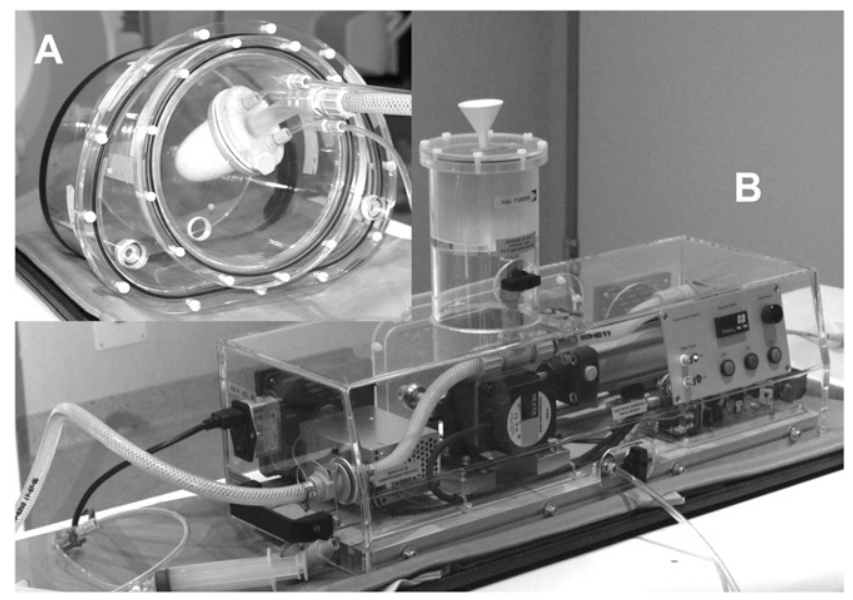

FIGURE 1. Elements of cardiac phantom used in this study. (A) Torso-anthropomorphic phantom with dynamic insert simulating beating left ventricle. (B) External pump-control unit. steps). Because of the absence of extracardiac activity, the images of the thorax phantom had heart-to-background count ratios that were higher than those obtained in clinical scenarios but had comparable myocardial wall-to-ventricular cavity count ratios.

Tomographic reconstructions were performed on an E-Soft system workstation (Siemens Medical Systems, Inc.) at the Department of Nuclear Medicine and PET/CT Centre, Oncology Institute of Southern Switzerland, Bellinzona, Switzerland. Transaxial slices with a thickness of $6.59 \mathrm{~mm}$ were reconstructed with 3D OSEM-based protocols and postfiltered with a symmetric 3D gaussian function (17). Tomographic reconstructions were obtained with different parameters $(10,12,15$, and 20 iterations; 4, 8, and 16 subsets; and FWHM filter cutoff values of 8, 10, 12, and $15 \mathrm{~mm}$ ) to determine the best combination for the correct estimation of the phantom parameters (Fig. 2). In OSEM reconstructions, the effects of subset and iteration numbers should be additive over the image characteristics (18). Thus, for simplification purposes, we chose to describe the different ( $I$ and $S$ ) combinations by using the expectation maximization-equivalent (EM-equivalent) iterations (defined as the product of iterations times subsets $[I \times S$ product $])$. The same angles for cardiac reorientation were maintained during all reconstruction processes.

The standard QGS program (version 2007-0.0.6.5.9.1) was used to measure the cardiac volumes and estimate the ejection fraction from each set of reconstructed gated images. The EDV, ESV, SV, and LVEF were automatically calculated. To better analyze the effects of the various combinations of OSEM parameters on the characteristics of the reconstructed images, we estimated the noise level in the myocardial wall and the contrast obtained between the ventricular wall and cavity as follows.

\section{Noise}

The mean myocardial counts $(M)$ and corresponding SDs $(S D)$ were determined from a ring-shaped, 3-pixel-thick region of interest (ROI) placed over the myocardium. Noise was assessed as the percentage root-mean-square $[100 \times(S D / M)](18)$. The same midventricular end-diastolic (frame 1) short-axis slice was selected for each set of reconstructed data.

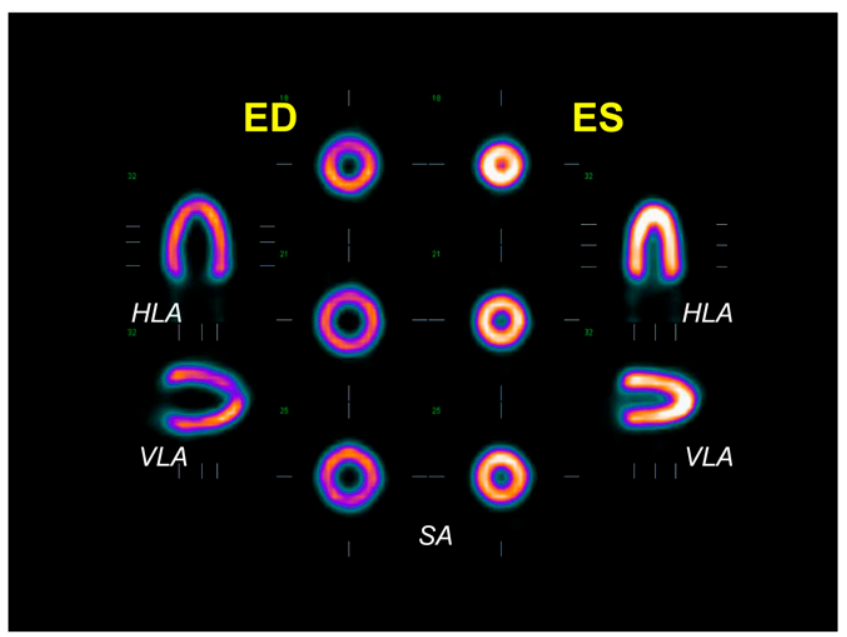

FIGURE 2. End-diastolic (ED) and end-systolic (ES) representative tomographic slices reoriented along major cardiac axes (short axis [SA], vertical long axis [VLA], and horizontal long axis [HLA]) obtained from reconstruction of G-SPECT phantom study with 3D OSEM iterative protocol. 


\section{Contrast}

On the same midventricular end-diastolic (frame 1) short-axis slice, a second ROI $(3 \times 3$ pixels $)$ was drawn at the center of the ventricular cavity. The signal and background counts were defined as the average pixel values in the myocardial (ring-shaped) ROI and in the ventricular cavity ROI, respectively. Contrast was defined as (signal - background)/background.

The results were expressed as mean $\pm \mathrm{SD}$.

\section{RESULTS}

The number of EM-equivalent iterations of the various combinations of parameters ranged from $40(4 S \times 10 I)$ to $320(16 S \times 20 I)$. An increase in the number of EMequivalent iterations produced higher contrast and increased the noise level in the myocardial wall (Fig. 3). Contrast and noise showed an almost linear relationship over lower EM-equivalent iterations; this relationship became quite flat at higher EM-equivalent iterations.

The LV function results obtained with the QGS software demonstrated a systematic underestimation of the LV volumes, with values ranging from 72 to $92 \mathrm{~mL}$ (differences of $-36 \%$ to $-18 \%$ with respect to the true volumes) for $\mathrm{EDV}$, from 18 to $32 \mathrm{~mL}$ (differences of $-51 \%$ to $-14 \%$ ) for $\mathrm{ESV}$, and from 54 to $63 \mathrm{~mL}$ (differences of $-28 \%$ to $-16 \%$ ) for SV. The plot of the measures of the 3 volumes revealed a bell-shaped curve, with higher values at the intermediate range of EM-equivalent iterations (80160), as shown in Figure 4. Within this range of EM-

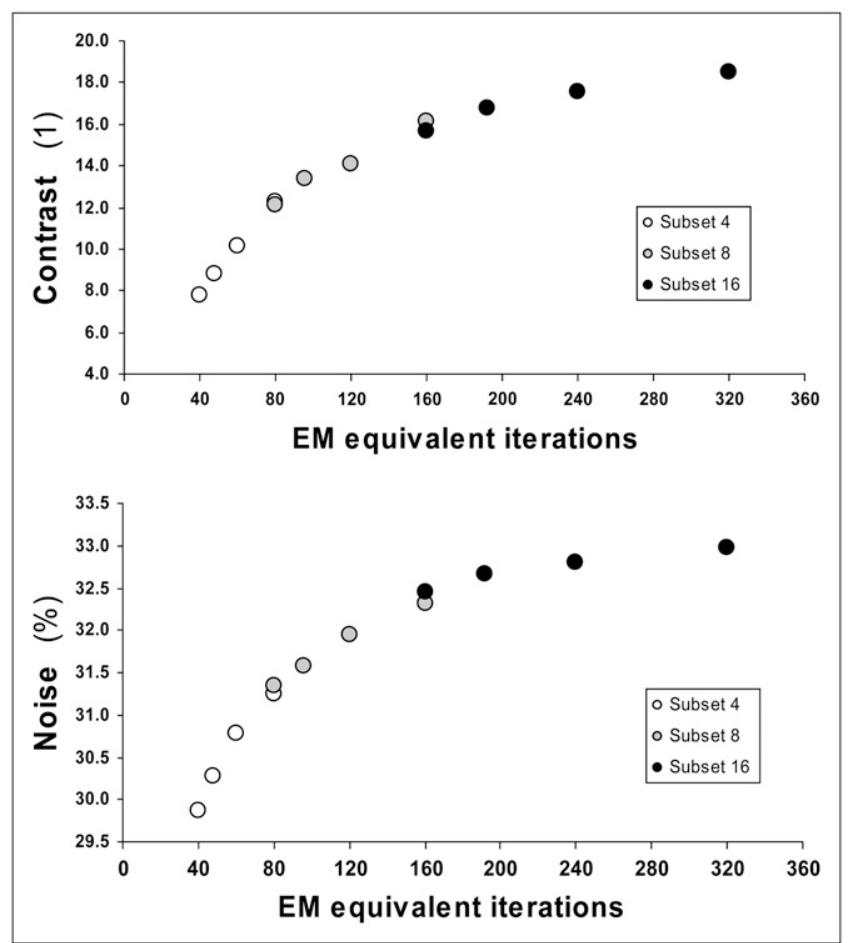

FIGURE 3. Measured contrast and noise for various EMequivalent iterations $(I \times S$ product) with fixed gaussian postprocessing filter FWHM value of $10 \mathrm{~mm}$. equivalent iterations, we found comparable results without important differences among the combinations (EDV, $91 \pm$ $2 \mathrm{~mL}$; ESV, $30.5 \pm 1.5 \mathrm{~mL} ; \mathrm{SV}, 60 \pm 1.7 \mathrm{~mL}$ ) and obtained a correct calculation of the $\operatorname{LVEF}(67 \% \pm 0.5 \%)$. The calculated LVEF ranged from $65 \%$ to $76 \%$ and was represented by a U-shaped curve with respect to the LV volumes, with lower central values and relative overestimations at the extremes of the EM-equivalent iterations used (Fig. 4).

The cutoff value for the gaussian filter significantly affected the underestimation of the cardiac volumes. FWHM values of 8 and $10 \mathrm{~mm}$ had similar effects, whereas increasing the FWHM values to 12 and $15 \mathrm{~mm}$ resulted in progressive reductions in the calculated LV volumes regardless of the combinations of $S$ and $I$ used (Fig. 5). Moreover, FWHM values exceeding $10 \mathrm{~mm}$ caused a clear overestimation of the LVEF (Fig. 6).

Overall, the combination of $10 \mathrm{I}, 8 \mathrm{~S}$, and a gaussian filter cutoff value of $10 \mathrm{~mm}$ produced the most accurate results, with an EDV of $89 \mathrm{~mL}$ (difference of $-20.5 \%$ with respect to the true value), an ESV of $29 \mathrm{~mL}$ (difference of $-21.6 \%$ ), an SV of $60 \mathrm{~mL}$ (difference of $-20 \%$ ), and an LVEF of $67 \%$ (no difference).

\section{DISCUSSION}

To our knowledge, this is the first study using a dynamic cardiac phantom to characterize a 3D OSEM iterative protocol for the estimation of LV function and LV volumes with ${ }^{99 m}$ Tc-labeled agent G-SPECT. A few comparative analyses of quantitative data obtained from defined OSEM protocols and FBP for clinical populations or obtained with other methods (such as cardiac MRI and radionuclide ventriculography) as standards of reference have been published, but no systematic analysis of the effects of OSEM reconstruction parameters in a dynamic cardiac phantom study had been performed yet (10-16).

We tested different combinations of $I$ and $S$ and postprocessing filter cutoff values to define the best protocol configuration for maximizing the accuracy of 3D OSEM in association with the QGS program for calculation of the LVEF and LV volumes. Our results showed that after GSPECT reconstruction with optimized 3D OSEM, the QGS program was able to correctly calculate the LVEF of the cardiac phantom but provided an underestimation of the LV volumes of about $-20 \%$. The data demonstrated that the underestimation decreased progressively with the initial increase in EM-equivalent iterations and then the underestimation increased again for higher values (EM-equivalent iterations exceeding 160), as depicted by the bell-shaped distribution of the estimated values. This behavior can be explained by the interactions between the different levels of contrast and noise of the images and the QGS algorithm of myocardial contour detection.

As the EM-equivalent iterations increased, they resulted in a progressive improvement in the contrast between the 


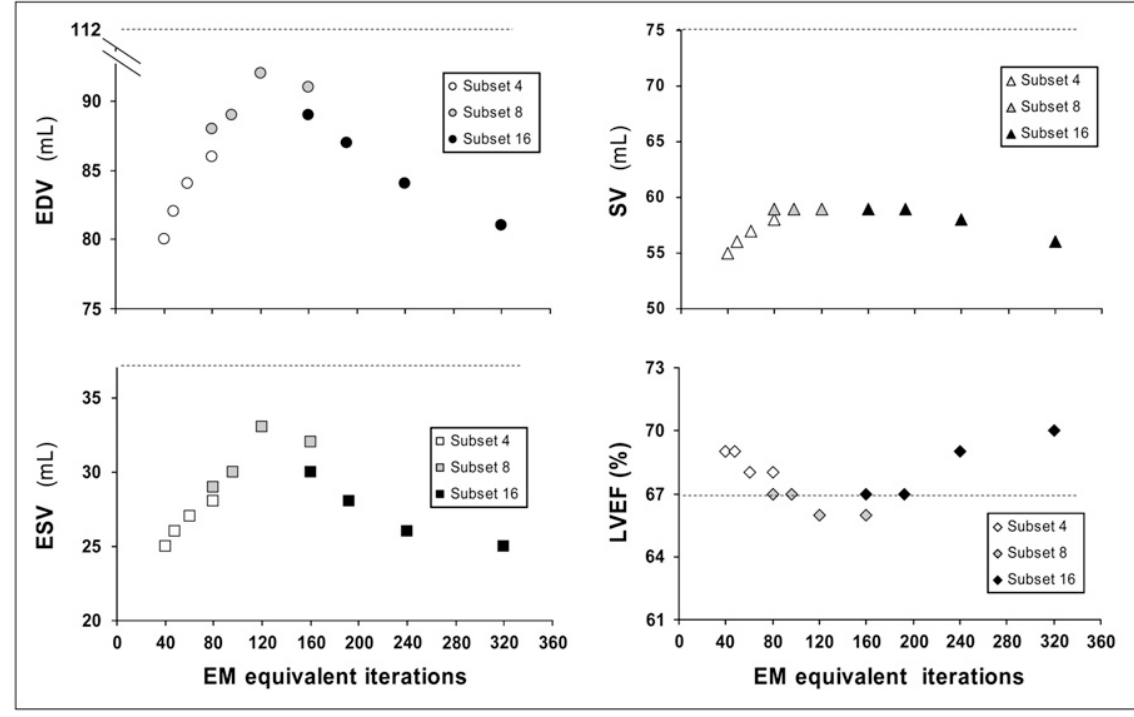

FIGURE 4. Measured EDV, ESV, SV, and corresponding LVEF with various EM-equivalent iterations $(I \times S$ product) and fixed gaussian postprocessing filter FWHM value of $10 \mathrm{~mm}$. Dotted lines represent actual values for EDV, ESV, SV, and LVEF of cardiac phantom. myocardial wall and ventricular cavity and a decrease in spatial blur. Nevertheless, at the same time, statistical noise in the count distributions in the ventricular wall grew. The higher statistical noise led to more indeterminate count profiles in the ventricular wall, causing an increase in the width of the bell-shaped gaussian fitting applied in the QGS program to identify the wall thickness and the ventricular edges. This factor resulted in a shift between estimated and actual endocardial edges that produced the underestimation for the ventricular cavity $(19,20)$. On the other hand, the bell-shaped distribution of the calculated ventricular volumes suggested that for EM-equivalent iterations ranging from 80 to 160, the opposite effects of noise and contrast seemed to be balanced and induced only slight changes in the QGS measurements.

The growing noise level due to the increasing $S$ and $I$ values can be corrected by postfiltration; we used a gaussian 3D postreconstruction filter because this was the only

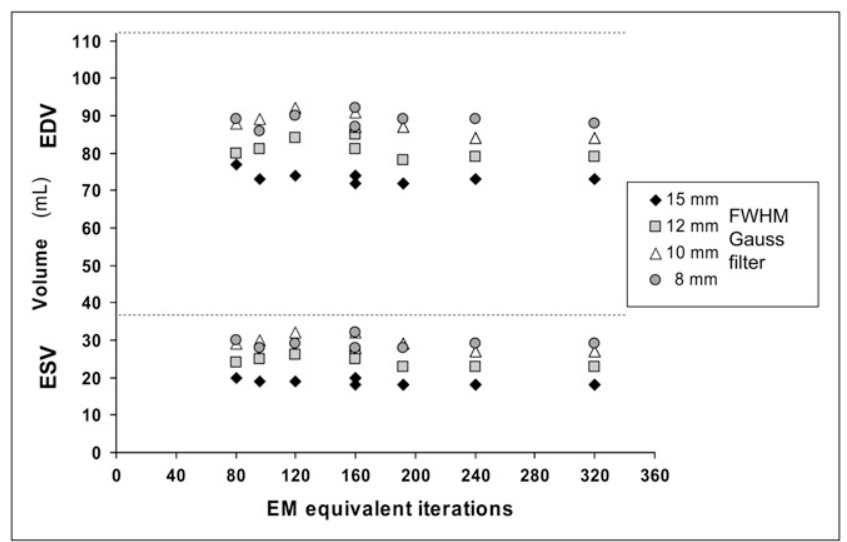

FIGURE 5. Measured LV volumes (EDV and ESV) with different gaussian postprocessing filter FWHM values for various EM-equivalent iterations $(I \times S$ product). Dotted lines represent actual values for EDV and ESV of cardiac phantom. choice available in our software system. Postfiltration had a strong impact on the calculated LV volumes at FWHM values exceeding $10 \mathrm{~mm}$, bringing about an increase in the underestimation for all the $I \times S$ combinations used in 3D OSEM. This effect is a clear consequence of the cutoff of the higher spatial frequencies that, reducing noise, reintroduced blur in the images, deteriorating the myocardial edge definition by the LV function quantification algorithms. Therefore, the type and level of postfiltration must be considered to be integral parts of the 3D OSEM algorithm applied.

A similar underestimation of LV volumes has been demonstrated in other cardiac phantom studies performed with standard or optimized FBP protocols (with Butterworth and Wiener prefiltration) and the QGS program $(21,22)$. These findings have also been confirmed in different clinical studies comparing ${ }^{99 \mathrm{~m}} \mathrm{Tc}$-labeled agent G-SPECT reconstructed with FBP and quantified with the

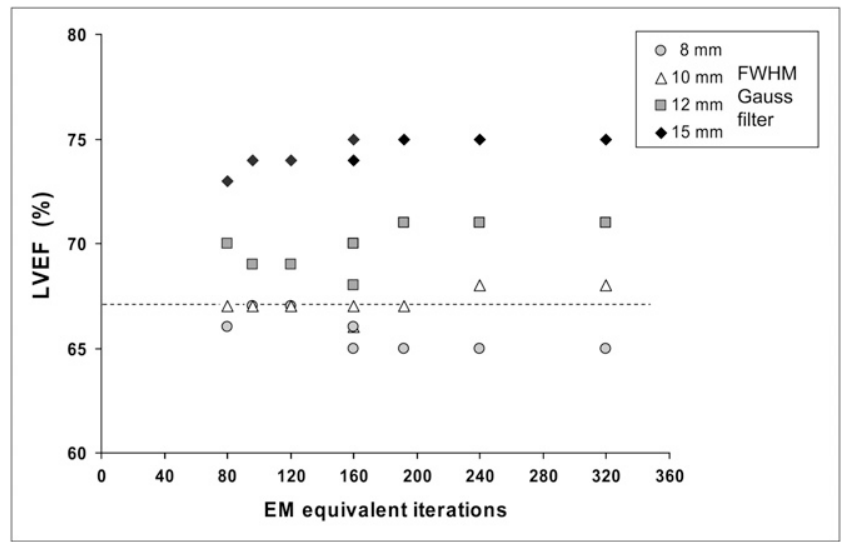

FIGURE 6. Calculated LVEF with different gaussian postprocessing filter FWHM values for various EM-equivalent iterations $(I \times S$ product). Dotted line represents actual value for LVEF of cardiac phantom. 
QGS program, contrast left ventriculography, and MRI (23-26).

Few data on OSEM filters have been published. Daou et al. (16) found a systematic underestimation of the LV volumes with OSEM ( $8 S$ and $2 I=16$ EM-equivalent $I$ ), the QGS program, and ${ }^{201} \mathrm{Tl}$ G-SPECT relative to left ventriculography, but they found comparable values for the LVEF. The same authors reported an increase in the LV volumes after the introduction into the iterative protocol of a depth-dependent resolution recovery algorithm without a significant modification of the LVEF.

Marie et al. (15) obtained a comparable underestimation of the LV volumes with ${ }^{99 \mathrm{~m}} \mathrm{Tc}$ sestamibi G-SPECT relative to cardiac MRI. In that study, the same OSEM protocol ( $8 \mathrm{~S}$ and $2 I$, with a depth-dependent resolution recovery algorithm) was tested with temporal Fourier filtering, and similar lower LV volumes were obtained with G-SPECT than with MRI. However, apart from the different strategies used for reconstruction, the underestimation of the LV volumes generally occurring with G-SPECT seemed to be related to the intrinsic limit of SPECT spatial resolution, which does not allow accurate estimation of the absolute values for thickness and thickening of the myocardial wall (usually $<20 \mathrm{~mm}$ ) and, therefore, to a lesser degree, correct LV volume measurements, especially in small left ventricles.

We used an 8-frame G-SPECT acquisition; this solution, which is largely applied in clinical practice because of the better count statistics with respect to the higher number of frames, may have further affected the underestimation of the LV volumes. Some published studies demonstrated that a temporal sampling of at least 16 frames per cardiac cycle is needed for accurate LVEF and LV volume measurements, particularly with the QGS program $(25,27-$ 30). Nevertheless, human studies based on the reframing of G-SPECT images acquired with higher framing rates have produced conflicting results. A phantom study did not demonstrate a clear superiority of a 16-frame acquisition over an 8-frame acquisition, with a similar underestimation of the LV volumes and a slight overestimation of the LVEF with the 16-frame solution (21). Moreover, a recent study comparing the quantitative data obtained with the QGS program from the 2 framing solutions (8 frames and 16 frames) acquired simultaneously showed only minor differences of questionable clinical value (31).

High correlations have been demonstrated in comparative studies of the LV volumes and LVEF estimates obtained by different methods of quantification, particularly in patients with a normal-size hearts $(25,32,33)$. Nevertheless, because of inherent algorithm differences, it has been suggested that the interchangeable use of different software packages should be avoided. Likewise, although the effects of the OSEM protocols on the characteristics of images were the same, the parameters were optimized for the QGS program and therefore should be applied with caution to other programs for quantification. A dedicated study should be performed for each program.

\section{CONCLUSION}

Our phantom study showed that the QGS program was able to correctly estimate the LVEF when used in association with an optimized 3D OSEM algorithm but underestimated by about $20 \%$ the LV volumes. The 3D OSEM protocol showed the best overall performance when applied with 8 subsets, 10 iterations, and a gaussian function FWHM value of $10 \mathrm{~mm}$. However, various combinations of technical parameters, such as an intermediate number of EM-equivalent iterations (80-160) and an FWHM value of less than or equal to $10 \mathrm{~mm}$ for gaussian postfiltration, allowed similar accurate results without clinically relevant differences in the LV volumes and the LVEF estimates.

\section{ACKNOWLEDGMENTS}

We are greatly indebted to Gilles Allenbach, Luca Modolo, and Didier Maillard for performing the phantom acquisitions. This study was supported by an Academic Research Award from the Leenaards Foundation, Lausanne, Switzerland.

\section{REFERENCES}

1. Blocklet D, Seret A, Popa N, Schoutens A. Maximum-likelihood reconstruction with ordered subsets in bone SPECT. J Nucl Med. 1999;40:1978-1984.

2. Case JA, Licho R, King MA, Weaver JP. Bone SPECT of the spine: a comparison of attenuation correction techniques. J Nucl Med. 1999;40:604-613.

3. Kauppinen T, Koskinen MO, Alenius S, Vanninen E, Kuikka JT. Improvement of brain perfusion SPECT using iterative reconstruction with scatter and nonuniform attenuation correction. Eur J Nucl Med. 2000;27:1380-1386.

4. Vanhove C, Defrise M, Franken PR, Everaert H, Deconinck F, Bossuyt A. Interest of the ordered subsets expectation maximization (OS-EM) algorithm in pinhole single-photon emission tomography reconstruction: a phantom study. Eur J Nucl Med. 2000;27:140-146.

5. Wells GR, King MA, Simkin PH, et al. Comparing filtered backprojection and ordered-subsets expectation maximization for small-lesion detection and localization in ${ }^{67} \mathrm{Ga}$ SPECT. J Nucl Med. 2000;41:1391-1399.

6. Hesse B, Tägil K, Cuocolo A, et al. EANM/ESC procedural guidelines for myocardial perfusion imaging in nuclear cardiology. Eur J Nucl Med Mol Imaging. 2005;32:855-897.

7. Bai J, Hashimoto J, Suzuki T, et al. Comparison of image reconstruction algorithms in myocardial perfusion scintigraphy. Ann Nucl Med. 2001;15:79-83.

8. Narayanan MV, King MA, Pretorius PH, et al. Human-observer receiveroperating-characteristic evaluation of attenuation, scatter, and resolution compensation strategies for ${ }^{99 \mathrm{~m}} \mathrm{Tc}$ myocardial perfusion imaging. J Nucl Med. 2003;44:1725-1734.

9. Hutton RL, Hutton BF, Angelides S, Choong KK, Larcos G. Improved tolerance to missing data in myocardial perfusion SPECT using OSEM reconstruction. Eur J Nucl Med Mol Imaging. 2004;31:857-861.

10. LaCroix KJ, Tsui BMW, Frey EC, Jaszczak RJ. Receiver-operating-characteristic evaluation of iterative reconstruction with attenuation correction in ${ }^{99 \mathrm{~m}} \mathrm{Tc}-$ sestamibi myocardial SPECT images. J Nucl Med. 2000;41:502-513.

11. Gilland DR, Tsui BM, Metz CE, Jaszczak RJ, Perry JR. An evaluation of maximum likelihood-expectation maximization reconstruction for SPECT by ROC analysis. J Nucl Med. 1992;33:451-457.

12. Ficaro EP, Fessler JA, Shreve PD, Kritzman IN, Rose PA, Corben JR. Diagnostic accuracy of attenuation-corrected ${ }^{99 \mathrm{~m}} \mathrm{Tc}$-sestamibi single-photon emission computed tomography. Circulation. 1996;93:463-473.

13. Kêdziora K, Lass P. Does quantification of myocardial perfusion SPECT study differ while image reconstruction is carried out using iteration algorithm instead of filtered back-projection? Preliminary report. Nucl Med Rev Cent East Eur. 2001;4:93-96.

14. Pretorius PH, King MA, Gifford HC, et al. Myocardial perfusion SPECT reconstruction: receiver operating characteristic comparison of $\mathrm{CAD}$ detection accuracy of filtered backprojection reconstruction with all of the clinical imaging 
information available to readers and solely stress slices iteratively reconstructed with combined compensation. J Nucl Cardiol. 2005;12:284-293.

15. Marie PY, Djaballah W, Franken PR, et al. OSEM reconstruction, associated with temporal Fourier and depth-dependant resolution recovery filtering, enhances results from sestamibi and ${ }^{201} \mathrm{Tl}$ 16-interval gated SPECT. J Nucl Med. 2005;46: 1789-1795.

16. Daou D, Pointurier I, Coaguila C, et al. Performance of OSEM and depthdependent resolution recovery algorithms for the evaluation of global left ventricular function in ${ }^{201} \mathrm{Tl}$ gated myocardial perfusion SPECT. J Nucl Med. 2003;44:155-162.

17. Beekman FJ, Viergever MA. Evaluation of fully 3D iterative scatter compensation and post-reconstruction filtering in SPECT. In: Grangeat P, Amans J-L, eds. ThreeDimensional Image Reconstruction in Radiology and Nuclear Medicine. Dordrecht, The Netherlands: Kluwer Academic Publishers; 1996:163-175.

18. Brambilla M, Cannillo B, Dominietto M, Leva L, Secco C, Inglese E. Characterization of ordered-subsets expectation maximization with 3D postreconstruction Gauss filtering and comparison with filtered backprojection in 99mTc SPECT. Ann Nucl Med. 2005;19:75-82.

19. Achtert AD, King MA, Dahlberg ST, Pretorius PH, LaCroix KJ, Tsui BM. An investigation of the estimation of ejection fractions and cardiac volumes by a quantitative gated SPECT software package in simulated gated SPECT images. J Nucl Cardiol. 1998;5:144-155.

20. Ford PV, Chatziioannou SN, Moore WH, Dhekne RD. Overestimation of the LVEF by quantitative gated SPECT in simulated left ventricles. J Nucl Med. 2001;42:454-459.

21. Visser JJN, Sokole EB, Verberne HJ, et al. A realistic 3-D gated cardiac phantom for quality control of gated myocardial perfusion SPECT: the Amsterdam gated (AGATE) cardiac phantom. Eur J Nucl Med Mol Imaging. 2004;31:222-228.

22. Kubo N, Mabuchi M, Katoh C, et al. Accuracy and reproducibility of left ventricular function from quantitative, gated, single photon emission computed tomography using dynamic myocardial phantoms: effect of pre-reconstruction filters. Nucl Med Commun. 2002;23:529-536.

23. Yoshioka J, Hasegawa S, Yamaguchi H, et al. Left ventricular volumes and ejection fraction calculated from quantitative electrocardiographic-gated ${ }^{99 \mathrm{~m}} \mathrm{Tc}-$ tetrofosmin myocardial SPECT. J Nucl Med. 1999;40:1693-1698.

24. Abe M, Kazatani Y, Fukuda H, Tatsuno H, Habara H, Shinbata H. Left ventricular volumes, ejection fraction, and regional wall motion calculated with gated technetium-99m tetrofosmin SPECT in reperfused acute myocardial infarction at super-acute phase: comparison with left ventriculography. $\mathrm{J} \mathrm{Nucl}$ Cardiol. 2000;7:569-574.

25. Lipke CS, Kühl HP, Nowak B, et al. Validation of 4D-MSPECT and QGS for quantification of left ventricular volumes and ejection fraction from gated ${ }^{99 \mathrm{~m}} \mathrm{Tc}-$ MIBI SPECT: comparison with cardiac magnetic resonance imaging. Eur J Nucl Med Mol Imaging. 2004;31:482-490.

26. Bax JJ, Lamb H, Dibbets P, et al. Comparison of gated single-photon emission computed tomography with magnetic resonance imaging for evaluation of left ventricular function in ischemic cardiomyopathy. Am J Cardiol. 2000;86:12991305 .

27. Germano G, Kiat H, Kavanagh PB, et al. Automatic quantification of ejection fraction from gated myocardial perfusion SPECT. J Nucl Med. 1995;36:2138-2147.

28. Manrique A, Faraggi M, Vera P, et al. 201-Tl and ${ }^{99 \mathrm{~m}}$ Tc-MIBI gated SPECT in patients with large perfusion defects and left ventricular dysfunction: comparison with equilibrium radionuclide angiography. J Nucl Med. 1999; 40:805-809.

29. Manrique A, Faraggi M, Vera $P$, et al. Effect of temporal sampling of left ventricular ejection fraction by means of thallium-201 gated SPECT: comparison of 16- amd 8-interval gating, with reference to equilibrium radionuclide angiography. Eur J Nucl Med. 2000;27:694-699.

30. Faber TL, Vansant JP, Pettigrew RI, et al. Evaluation of left ventricular endocardial volumes and ejection fractions computed from gated perfusion SPECT with magnetic resonance imaging: comparison of two methods. $\mathrm{J} \mathrm{Nucl}$ Cardiol. 2001;8:645-651.

31. Montelatici G, Sciagrà R, Passeri A, Dona M, Pupi A. Is 16-frame really superior to 8-frame gated SPECT for the assessment of left ventricular volumes and ejection fraction? Comparison of two simultaneously acquired gated SPECT studies. Eur J Nucl Med Mol Imaging. 2008;35:2059-2065.

32. Schaefer WM, Lipke CS, Standke D, et al. Quantification of left ventricular volumes and ejection fraction from gated ${ }^{99 \mathrm{~m} T c-M I B I}$ SPECT: MRI validation and comparison of the Emory Cardiac Tool Box with QGS and 4D-MSPECT. J Nucl Med. 2005;46:1256-1263.

33. Hambye AS, Vervaet A, Dobbeleir A. Variability of left ventricular ejection fraction and volumes with quantitative gated SPECT: influence of algorithm, pixel size and reconstruction parameters in small and normal-sized hearts. Eur J Nucl Med Mol Imaging. 2004;31:1606-1613. 